\title{
Time and information in the foundations of physics
}

\author{
Vasil Penchev, vasildinev@gmail.com \\ Bulgarian Academy of Sciences: Institute of Philosophy and Sociology: \\ Dept. of Logic and Philosophy of Science
}

\begin{abstract}
The paper justifies the following theses: The totality can found time if the latter is axiomatically represented by its "arrow" as a well-ordering. Time can found choice and thus information in turn. Quantum information and its units, the quantum bits, can be interpreted as their generalization as to infinity and underlying the physical world as well as the ultimate substance of the world both subjective and objective. Thus a pathway of interpretation between the totality via time, order, choice, and information to the substance of the world is constructed. The article is based only on the well-known facts and definitions and is with no premises in this sense. Nevertheless it is naturally situated among works and ideas of Husserl and Heidegger, linked to the foundation of mathematics by the axiom of choice, to the philosophy of quantum mechanics and information.
\end{abstract}

Key words: choice, order, quantum information, time, totality, well-ordering 
The objectivity of the paper is to discuss time as if crucified on the cross figuratively situated between subject and object therefore unifying them. On the one hand, time is the foundation of consciousness. On the other hand, it is the basis shared by the physical world. Furthermore, the link between the conscious and physical realized by time is an ordering having two faces, from which the one addresses choice, and the other continuity. The unification of both represents a unit of information, which also unifies subject and object.

That task comprises the research of the following questions:

1. Can the true totality found time as a well ordering?

2. How can time found choice?

3. How can choice found information?

4. Can information underlie the physical world as the general substance?

5. Is the totality the true and ultimate substance of the world both objective and subjective?

Quantum mechanics, mathematics, and philosophy are the areas, in which one can search for answers.

\section{Can the true totality found time as a well ordering?}

Time can be exhaustedly described and thus axiomatically defined by its most essential property: the "arrow" of time. Indeed time serves to separate and order well the world both subjective and objective in a rigorous sequence known in mathematics as "well-ordering". It means that the following conditions should be satisfied:

The axiom of trichotomy, if a moment of time is given, any other should be either (1) past or (2) future in relation to it or (3) coincide with it.

Any period of time has a beginning, i.e. an initial moment of time.

Described and defined in thus, the totality can found time as follows:

The totality possesses the following paradoxical and definitive property: It contents necessarily its externality being just "total". However it contents its internality, the intersection of which with the externality of totality is an empty set or at least does not coincide with the internality in general. This implies that the totality should possess special elements which can be defined by the controversial property that they do not belong to its internality. Consequently, the totality generates necessarily a well-ordering such as a set of sets, any of which is a subset of another of those subsets. 
Indeed that set satisfies the axiom of trichotomy: If an element of it is given, any other is either (1) a true subset of it or (2) contents it as a true subset, or (3) coincides with it.

Any subset of that set possesses an initial, or in other words, maximal element, to which all others are subsets.

The well-ordering generated in thus is equivalent to time as it has been defined above only by its arrow, i.e. as any well-ordering.

In particular, this implies that the totality can be only infinite for any finite set can constitute only a finite series and reaching the empty set, which is ultimate, it cannot include its externality within itself.

That totality discussed above as generating time as a well-ordering can be interpreted both subjectively (as consciousness) or objectively (as the universe) leading correspondingly to subjective time in the former case or to objective time in the latter one. However the underlying structure does not depend of whether the interpretation is subjective thinking of the totality as consciousness or objective as the universe. Thus time being the same in both cases can unify subject and object as two equivalent interpretations of the totality. There exists a fundamental philosophical invariance of subject and object expressed by time and originating from the totality.

\section{How can time found choice?}

After time has seen as well-ordering, and any series of sequentially nested sets is a well-ordering, the next questions are: Does any well-ordering imply at least one sequence of nested sets? Does choice can be represented in terms of set theory as a set nested in another, i.e. as the pair of a set and its subset? Does any sequence of nested sets and thus a well-ordering can be interpreted as an equipotent series of choices? Does well-ordering and choice are equivalent concepts in a sense? Furthermore, if these three: well-ordering, choice and a sequence of nested sets are given as equivalent, do these structures imply back time in turn and by means of it, the totality?

The answers of all questions are positive and can be found as follows:

Any well-ordering implies at least one sequence of nested sets because one can construct the later on the base of the former: The well-ordering is a well-ordered set, which is taken as the initial member of the searched sequence. Then any next element of it can be obtained from the previous one by removing of an element.

Then choice can be defined by two sets: the one is that of all possible alternatives, and the other is the subset of the chosen alternative. If that subset is a true subset of the set of all alternatives, the corresponding choice will be designated as true, too. 
Obviously, this definition of choice can applied for any sequence of choices therefore generating an equivalent well-ordering or a sequence of nested sets. One can generalize the definition of choice maximally as an always admissible option of choice. That generalization is well-known in mathematics as the axiom of choice, and in philosophy, too, as freewill ordinarily discussed in relation to human beings or their rights. That choice, which is always allowed, implies a wellordering in any case, and vice versa as above. This statement is known in mathematics as the equivalence of the well-ordering theorem and the axiom of choice. It can be extended in relation to freewill coined in an abstract and generalizing sense to the being at all rather than the human being only.

The cited equivalence in mathematics and eventually in philosophy can be interpreted as implying or originating from the equivalence of choice and order. The concept of information can be founded both mathematically and philosophically on that equivalence unifying these two fundamental ideas. This will be discussed a little below.

At last, that generalizing choice being equivalent both to a sequence of nested sets and to a wellordering turns out to be equivalent to time as far as it has defined axiomatically as well-ordering. If the totality implies time in this sense, does the well-ordering or time implies the totality in turn? Taking an infinite series of embedded sets equivalent both to time and to choice, one can interpreted as a well-ordered set of externalities of the totality contained by it and therefore implying it. Thus a bidirectional pathway between choice and the totality is already in exploitation guaranteeing the reliable link of equivalence between them across the border between subject and object without any dependence on any of them.

\section{How can choice found information?}

Information is order in a most general sense. After order is linked to choice as above, this connects also choice and information indirectly. However, there is another, unmediated relation between them founded on the quantity of information: Its unit is an elementary choice, a binary digit, a bit. Indeed a bit is the choice between two equiprobable alternatives and thus the quantity of information indicates the amount of choice of a set in units of elementary choice. That amount of information of a set can be also interpreted equivalently as its complexity, i.e. as that number of elementary operations, by which it can be ordered beginning from an absolute chaos, or in other words, in how many of elementary steps it can be constructed from nothing, from zero, i.e. from the empty set.

That foundation of information on choice (or order) refers only to finite sets and it does not require the axiom of choice (or the well-ordering theorem). However if one utilizes the latter two, the concept of information can be extended to any infinite set. Then the unit of information should be relevantly generalized as a choice among an infinite set of alternatives, which can be always enumerated once the axiom of choice is admitted. That unit might be designated as an 
"init" for an "infinite digit" just as a bit is for a "binary digit", and the term of "information" might be interpreted as "infinite formation". However they are already introduced under other names and for other reasons, which will be discussed in detail in the paragraph 4 . Both the term of init and the interpretation of information as infinite formation will be kept here to be conserved the logical consequence. Furthermore, "qubit" and "quantum information", which will be introduced below, can be considered only as particular cases correspondingly of "init" and "infinite information" (or "in-formation") thus questioning about their eventual equivalence.

After involving the axiom of choice for introducing "init" and "in-formation", some ostensibly paradoxical corollaries are inevitable. They are due to involving infinity in final analysis, which is wrongly interpreted in terms of finiteness. More exactly, infinity is able to be both universal (and thus complete) and open (and thus incomplete) unlike finiteness, which is always either complete or incomplete disjunctively. For example, a single "init" is equivalent to any, including infinite, number of inits. However that property is not controversial and it implies no contradictions or vagueness. A rigorous and quantitative theory of infinity can be (and has in fact already) developed under other name: quantum mechanics.

Another question is yet open: How does the introduction of init and in-formation affect the understanding of time and the totality for the latter two are equivalent to choice as this is investigated above? After the choice has considered as infinite in general, does this influence somehow the concepts of time and the totality?

The totality and thus time can be only infinite as this is deduced above. If the choice and thus information is finite, they cannot refer directly to the totality and time. After they have involved infinity in themselves, they are already able to represent or address the totality and time in an unmediated way. In other words, the concept of in-formation continues and thus keeps the bilaterality (bidirectionality) of the pathway between information and the totality in the newly built section from choice to information.

\section{Can information underlie the physical world as the general substance?}

The next questions are: If the "init" and "in-formation" are given correspondingly as the unit of an infinite choice and the quantity of those infinite choices necessary to be formed some structure, can the "qubit" and "quantum information" be deduced or even constructed from the former two? Do the latter and thus the former two underlie the physical world and even the world at all?

However, if the pathway from the totality to quantum information and thus to the world can be continued in the section from "in-formation" to quantum information, is it bilateral, bidirectional, i.e. are "in-formation" and quantum information equivalent to each other?

The answers of these new questions are also positive and can be justified as follows: 
The first question can be divided into two parts: The first one is: Is an element of an arbitrary set equivalent to the choice of the element of the set supplied by a metric structure? Then the next one would be: Is that choice equivalent to a qubit? If the answers of both questions would be positive, an "init" would be also equivalent to a qubit and the quantities measured by each of both measures, correspondingly "in-formation" and quantum information could always coincide.

Then one can start with the second half of the question. The choice of a point in a metric space means that exist some mapping, which depicts the set of all possible choices (i.e. of all points in the metric space) into the set of real numbers, and as the concept of choice implies the axiom of choice as above, into the set of natural numbers in final analysis. In other words, the restriction of the choice to a set having some metric structure is equivalent to the fact that the set of all possible choices is countable, and this is an obvious tautology as the concept of choice implies the axiom of choice and thus the countability of any set including that of all choices possible under any additional conditions. As all those cases are equivalent to each other, one can choose one of them to represent any other. That special representative case is the choice of a point of the unit sphere (i.e. a point of the surface of a ball of radius 1) for considerations, which follow a little below. That choice of a point from the unit sphere is isomorphic to a qubit if the latter is defined as usual as follows:

$$
\begin{aligned}
& 1 \text { qubit }=\alpha|0\rangle+\beta|1\rangle ; \alpha, \beta \in C ;|\alpha|^{2}+|\beta|^{2}=1 ; \\
& |0\rangle,|1\rangle \text { are two orthogonal subspaces of Hilbert space, } \\
& \text { e.g. such as two successive axes of it: } e^{i n \omega}, e^{i(n+1) \omega}
\end{aligned}
$$

Thus an "init" is equivalently representable as two complex numbers, the sum of the modules of which is equal to 1 , i.e. as a qubit.

This result can be generalized therefore answering the first half of the question. In fact the "qubit" has chosen to represent any case of an "init" just for this reason. The sense of this first half of the question is whether the choice is "transparent" in principle, or in other words, whether the choice of an element changes it, i.e. whether the element before and after choice coincides, whether it is one and the same in both cases.

To be rigorously mathematically defined the state of an element before to be chosen in general, the axiom of choice should be excluded as it guarantees that it can be always chosen and thus any discussion about the state before to be chosen would pointless. In fact, the removing of this axiom is not only necessary, but also sufficient to represent generally any element of any set before to be chosen. This means that any constructive procedure for an element to be specified among the others cannot be admitted. Coining terms of quantum mechanics, the state of a set, from which an element cannot be separated in any way, will be designated as "coherent". 
This preliminary consideration already hints that the mapping of a coherent state into a wellordered state of a set should be discussed not only mathematically, but also philosophically. Science used to resolve the analogical problem in classical physics by the postulate of the knowability of the world. It admits subject and object to be equated in the process of knowledge therefore introducing a common time both of subject and of object. However this solution is already inapplicable for the question refers to the relation of the absence and availability of choice, well-ordering and thus time at all making impossible for time to be premised as it should be deduced.

The introduction of the totality, from which time to be deduced, offers another approach. Indeed the totality has been considered as equivalent to time until now in the paper. This has been done after the division of its externality and internality had been admitted implicitly. However the totality should be thought as some oneness, which does not allow of any division into parts, e.g. these of the externality and of the internality. It should unify even its oneness and any division of it into any parts, to be total in fact. Consequently the relation between the totality and time, which represents well-ordering and thus choice and some division for the choice to make sense, is rather extraordinary: The totality both represents absolutely itself in the time and transcends it e.g. by its wholeness or by the doubling of time in eternity, etc.: The totality is absolutely expressed in the time, and nevertheless it includes one more thing inexplicable.

All this seems to be controversial, even contradictory, and thus both unbuildable and unembeddable in any mathematical structure. In fact, Hilbert space utilized by quantum mechanics is that structure. It coincides with its dual space, which cannot be simultaneously given with it. In other words, Hilbert space is absolutely represented by any member from the pair of dual spaces, and anyway that representation excludes something inexplicable, namely the other identical "twin" or counterpart. This initially contradictory ostensibly structure runs through all quantum mechanics generating e.g. many alleged “paradoxes”, “incompleteness”, etc. All of them originate from infinity involved in the core of the mathematical formalism of quantum mechanics for Hilbert space is infinitely dimensional and this fact is necessary to be resolved the main problem of how quantum leaps and smooth motions to be represented uniformly.

Indeed the totality is necessarily infinite, and infinity can be defined as what contains a true part of it, which is equivalent to it. Thus the complement of this true part, which is always nonempty, is an inexplicable remainder after any explication by any infinite part of. That division of infinity and thus of the totality is what is represented by the original invariance to the axiom of choice: The remainder inexplicable in principle is that, to which the axiom of choice should not be valid, and vice versa: What is explicable (in the time in final analysis) obeys the axiom of choice. The same can be thought as a special property of infinity or of the totality such as the reconciliation of universality and openness in them. 
All these considerations are not a rigorous deduction, but they cannot be that for any deduction, and logic in principle as well, is on the side of explication. Anyway one can generalize logic as ontology, which can involve the inexplicable in principle as above. Even more, that ontology including the inexplicable nevertheless allows a precious enough expression by a mathematical stricture such as Hilbert space, which can be thought as an extraordinary form of logic based on lattices in it. The above consideration should be a deduction within that logic.

All this should found the positive answer also of the first half of the question: Any set and even any entity can be equivalently represented by some metric structure admitting some inexplicable remainder, which does not decrease the exactness of that quantitative representation. The pair of the totality and time underlies ontologically that statement coinciding with it in a sense.

In fact any wave function can be represented as an equivalent series of qubits and thus as a value of the quantity of quantum information. Since that is the case, it underlies the physical world for any entity in it should be a quantum state representable as a wave function (a point in Hilbert space) or some change of that according to quantum mechanics. Consequently, the substance of the physical world is only quantum information and its change. This can be equivalently represented as the statement that the physical world is a single quantum computer processing just quantum information, and all physical processes are computations in it for quantum information is a generalization of information as to infinity. A quantum computer can be thought as a quantum Turing machine, in which all bits of its tape are replaced by qubits, and all operation on a cell (a qubit in the case) are the same: “Write!”, “Read!”, “Next!”, and “End!”

Even much more, not only the physical world, but the world at all can be considered as underlain by quantum information. The reason of that generalization is the invariance to object/ subject from the viewpoint of the totality and thus of time: Both interpretations of the totality as the universe being objective and the absolute (or transcendental) subject being subjective should be identical and that identity is expresses in particular by the availability of a common or joint foundation, quantum information, though the contemporary science has not yet managed to interpreted consciousness in terms of wave function directly.

If now one look at the "pathway" between the totality and quantum information globally, the newly built section between in-formation and quantum information involving explicitly infinity makes it to be rather extraordinary: like a Möbius strip, which is bilateral or bidirectional locally, but unilateral or unidirectional globally. In fact the Möbius strip embeds topologically the structure of a bit representing both its aspects as a single unit and as two disjunctive alternatives correspondingly globally and locally. One can imagine the totality topologically as that generalization of the Möbius strip, where infinitely many “sides” appear locally. 
Time or logic should be interpreted only locally after that visualization. That inexplicability featuring only the totality is seen by dint of the global viewpoint without complementing the "picture” by any additional detail: It is only a doubling completing it as a whole.

\section{Is the totality the true and ultimate substance of the world both objective and subjective?}

Thus quantum information can be very well understood as the local aspect or "hypostasis" of the totality being the true ultimate substance of the world both subjective and objective. One can think of quantum information as matter, i.e. as the absolutely explicable meaning of the substance therefore existing always in time.

Time unifying subject and object in turn is only the explicable "part" or "half" of the totality distinguishing from it or from all explicable by a "mute” or "ontological difference”: The totality is always both absolutely in time and yet transcending it by something inexplicable in principle by a pure or mirror doubling of all existing in time.

\section{Conclusions:}

Time can be defined axiomatically by its "arrow" therefore generalizing and representing the directionality of the world and being. Thus it can be equated to ordering and to the mathematical concept of well-ordering. That reduction can reveal its essential connection to choice and information.

As the well-ordered aspect of the world, it can be deduced from the totality involving necessarily infinity. The concept of choice and information can be relevantly generalized as to infinity and conserve their link to time even under the condition of infinity. That infinite information is equivalent to the notion of quantum information in quantum mechanics. Thus one can built a pathway between the totality and quantum information, which turns out to be locally bilateral and bidirectional but globally unilateral and unidirectional like a Möbius strip. Time can be seen as embodying this global aspect of the totality to be unidirectional and thus well-ordered, a quantum-informational structure.

In that scheme, the time expresses the totality absolutely and yet the totality includes some remainder always inexplicable in principle. The same scheme is also embedded in the basic mathematical structure of quantum mechanics: Hilbert space. Then its complementarity can be interpreted as that absolute expressibility always complemented by the inexplicable remainder of the incommeasurable twin of the conjugate quantity.

The totality can be interpreted as the ultimate substance of the world quantitatively representable as quantum information. Time is a unifying boundary between subject and object therefore corresponding to quantum information. 
Time offers a global viewpoint to the totality, from which can be seen both its completeness and openness contradicting common sense as it is locally related in principle. So the concept of time in philosophy allows of the totality as its subject to be described exactly enough and even more, to be directly linked to related notions in mathematics and experimental science, among which quantum mechanics is.

Any references were omitted purposely until now emphasizing the absence of premises necessary for that consideration. It can be deduced from commonly known facts and definitions mentioned where need be. That approach corresponds to the primary and initial nature both of time and the totality.

Nevertheless one can explicate a series of hidden, but obvious links to all corpus of contemporary cognition, especially in philosophy, mathematics, quantum mechanics and the theory of information and computation. They should be enumerated completely enough for the present text to be able to be sited in its relevant context enriching its meaning:

Those are works and ideas of Husserl (1905; 1911), Heidegger (1924; 1927; 1929a, b; 1956; 1957), and Derrida (1968).

A series of works on the foundation of mathematics elucidates the connection between the mathematical concepts of infinity, well-ordering and choice (Zermelo 1904; Skolem 1922; Jech 1973; Howard and Rubin 1998; Martin-Löf 2008). Furthermore one should estimate the revealing that infinity can unify universality, completeness and openness.

Quantum mechanics corroborates the absence of hidden variables in its area (Neumann 1932; Kochen and Specker 1968) and therefore the validity of the axiom of choice in an experimental science. Furthermore it involves the concept of entanglement and quantum correlations (Einstein et al. 1935; Schrödinger 1935; Bell 1964; Clauser and Horn 1974; Aspect et al. 1981; 1982), and thus quantum information, which can be thought as the substance of the physical world.

The theory of information clears up the close link between the concept of information (Shannon 1948; 1951) and those of choice and order (Kolmogorov 1965; 1968; Chatin 1966; 1969; 1975; 1977; 1987; Martin-Löf 1966). Its contemporary development as the theory of quantum information (for example: Wilde 2013) generalizes all of them as to infinity and allows of seeing how that quantum information, which is interpreted as infinite information, can underlie the physical world and the world at all. 


\section{References:}

Aspect A, Grangier R, Roger G (1981) Experimental tests of realistic local theories via Bell's theorem. Phys Review Lett 47: 460-463.

Aspect A, Grangier R, Roger G (1982) Experimental Realization of Einstein-Podolsky-RosenBohm Gedanken Experiment: A New Violation of Bell's Inequalities. Phys Rev Lett 49: 91-94.

Bell J (1964) On the Einstein - Podolsky - Rosen paradox. Physics (New York) 1: 195-200.

Chaitin G (1966) On the Length of Programs for Computing Finite Binary Sequences. J Assoc Comput Mach 13: 547-569

Chaitin G (1969) On the Simplicity and Speed of Programs for Computing Definite Sets of Natural Numbers. J Assoc Comput Mach 16: 407-412.

Chaitin G (1975) A Theory of Program Size Formally Identical to Information Theory. J Assoc Comput Mach 22: 329-340.

Chaitin G (1977) Algorithmic information theory. IBM J Res Dev 21: 350-359.

Chaitin G (1987) Algorithmic Information Theory. Cambridge University Press, Cambridge,

Clauser J, Horne M (1974) Experimental consequences of objective local theories. Phys Rev D 10: 526-535.

Derrida J (1968) Différance. In: Derrida J. Marges - de la philosophie. Éditions de Minuit, Paris, 1972, pp. 1-29.

Einstein A, Podolsky B, Rosen N (1935) Can Quantum-Mechanical Description of Physical Reality Be Considered Complete? Phys Rev 47: 777-780.

Heidegger M (1924) Der Begriff der Zeit. Gesamtausgabe (Hrsp F-W von Herrmann), Bd 64 (2004). Vittorio Klostremann, Frankfurt am Main.

Heidegger M (1927) Sein und Zeit. Gesamtausgabe (Hrsp F-W von Herrmann), Bd 2 (1977). Vittorio Klostremann, Frankfurt am Main.

Heidegger M (1929a) Kant und das Problem der Metaphysik. Gesamtausgabe (Hrsp F-W von Herrmann), Bd 3 (1991). Vittorio Klostremann, Frankfurt am Main.

Heidegger M (1929b) Vom Wesen des Grundes. Gesamtausgabe (Hrsp F-W von Herrmann), Bd 9 (1976). Vittorio Klostremann, Frankfurt am Main, pp 123-175.

Heidegger M (1956) Was ist die Zeit? Gesamtausgabe (Hrsp F-W von Herrmann), Bd 13 (1983). Vittorio Klostremann, Frankfurt am Main, p 131.

Heidegger M (1957) Identität und Differenz. Gesamtausgabe (Hrsp F-W von Herrmann), Bd 11 (2006), pp 27-110.

Howard P, Rubin, J (1998) Consequences of the Axiom of Choice. Mathematical Surveys and Monographs, vol 59. American Mathematical Society, Providence, R.I.

Husserl E (1905) Vorlesungen zur Phänomenologie des inneren Zeitbewußtfeins. Husserliana: Edmund Husserl - Gesammelte Werke (Hrsh R Boehm), Bd 10 (1966). Nijhoff (Springer) Dordrecht Boston London, pp 3-98. 
Husserl E (1911) Philosophie als strenge Wissenschaft. Husserliana: Edmund Husserl Gesammelte Werke (Hrsh R Boehm), Bd 25 (1987). Nijhoff (Springer) Dordrecht Boston London, pp 3-67.

Jech T (1973) The Axiom of Choice. Studies in Logic and the Foundations of Mathematics, vol 75, North-Holland Publ. Co., Amsterdam.

Kochen S, Specker E (1968) The problem of hidden variables in quantum mechanics. J Math Mech 17: 59-87.

Kolmogorov A (1965) Tri podkhoda k opredeleniyu "kolichestvo informatsii” (Three approaches to the definition of the quantity of information). Probl Inf Transm 1: 3-11.

Kolmogorov A (1968) Logical basis for information theory and probability theory. IEEE T Inform Theory IT-14: 662-664

Martin-Löf P (1966) The definition of random sequences. Inform Control 9: 602-619.

Martin-Löf P (2008) 100 years of Zermelo's axiom of choice: What was the problem with it? In: Lindström S et al (eds) Logicism, Intuitionism, and Formalism: What Has Become of Them? Synthese Lybrary, vol 341 (2009). Springer, Berlin Heidelberg New York, pp 209-219.

Neumann J (1932) Mathematische Grundlagen der Quantenmechanik. Verlag von Julius Springer, Berlin.

Schrödinger E (1935) Die gegenwärtige situation in der Quantenmechanik. Naturwissenschaften 48: 807-812; 49: 823-828; 50: 844-849.

Shannon C (1948) A Mathematical Theory of Communication. Bell Syst Tech J 27: 379-423.

Shannon C (1951) Prediction and entropy of printed English. Bell Syst Tech J 30: 50-64.

Skolem T (1922) Einige Bemerkungen zur axiomatischen Begründung der Mengenlehre. Selected works in logic (ed E Fenstad), 1970. Univforlaget, Oslo, pp 137-152.

Wilde M (2013) From Classical to Quantum Shannon Theory. Available via ARXIV. http://arxiv.org/abs/1106.1445 . Cited 26 Oct 2013.

Zermelo E (1904) Beweis, dass jede Menge wohlgeordnet werden kann. Math Ann 59: 514-16. 